\title{
Electromagnetic Radiation Efficiency of Body-Implanted Devices
}

\author{
Denys Nikolayev, ${ }^{1,2, *}$ Maxim Zhadobov, ${ }^{1}$ Pavel Karban, ${ }^{3}$ and Ronan Sauleau ${ }^{1}$ \\ ${ }^{1}$ Institut d'Électronique et de Télécommunications de Rennes (IETR \\ UMR CNRS 6164), University of Rennes 1, Rennes 35042, France \\ ${ }^{2}$ Imec - Ghent University, Ghent 9052, Belgium \\ ${ }^{3}$ Regional Innovation Centre for Electrical Engineering (RICE), \\ University of West Bohemia, Pilsen 30614, Czechia
}

(Dated: March 1, 2018)

\begin{abstract}
Autonomous wireless body-implanted devices for biotelemetry, telemedicine, and neural interfacing are an emerging technology providing powerful capabilities for medicine and clinical research. Here, we study the through-tissue electromagnetic propagation mechanisms, derive the optimal frequency range, and obtain the maximum achievable efficiency for radiative energy transfer from inside a body to free space. We analyze how polarization affects the efficiency by exciting TM and TE modes using a magnetic dipole and magnetic current source, respectively. Four problem formulations are considered with increasing complexity and realism of anatomy. The results indicate that the optimal operating frequency $f$ for deep implantation (the depth $d \gtrsim 3 \mathrm{~cm}$ ) lies in the $10^{8}-10^{9} \mathrm{~Hz}$ range and can be approximated as $f=2.2 \times 10^{7} / d$. For a subcutaneous case $(d \lesssim 3 \mathrm{~cm})$, the surface-waveinduced interference is significant: within the range of peak radiation efficiency (about $2 \times 10^{8} \mathrm{~Hz}$ to $3 \times 10^{9} \mathrm{~Hz}$ ), the $\mathrm{max} / \mathrm{min}$ ratio can reach a value of 6.5 . For the studied frequency range, $80-99 \%$ of radiation efficiency is lost due to the tissue-air wave-impedance mismatch. Parallel polarization reduces the losses by a few percent; this effect is inversely proportional to the frequency and depth. Considering the implantation depth, operating frequency, polarization, and directivity, we show that about an order-of-magnitude efficiency improvement is achievable compared to existing devices.
\end{abstract}

\section{INTRODUCTION}

Implantable, ingestible, and injectable (in-body) electronics offer breakthrough capabilities for medicine and clinical research [1]. Biotelemetry, for example, enables continuous monitoring of physiological parameters for disease diagnosis and prevention. Neural interfaces allow us to study the brain, to restore sensory function, and to assist in the rehabilitation of amputees, survivors of paralysis, and patients with neurodegenerative diseases [2, 3]. Continuous innovation in microelectromechanical systems, integrated circuits, and microfluidics drives the creation of novel applications. Likewise, wireless powering allows removing bulky batteries and avoiding going through surgery to replace them. It makes the lifespan of an implanted device practically unlimited. Kim et al. [4, 5] introduced a powering approach operating in mid field (the region where the wavelength is comparable to the distance between an implant and transmitter) and achieved much higher power transfer efficiency than traditional inductively coupled systems. Using this approach, Agrawal et al. [6] reported the conformal focusing system that makes it possible to power an ultra-miniature pacemaker, for instance [7].

Long-term operation of in-body devices relies on wireless technologies to interface with external systems. However, the electromagnetic (EM) energy transfer from a body into surrounding free space is generally inefficient. Extending the EM transmission range between a body

\footnotetext{
* Corresponding author. $\mathrm{d} @$ deniq.com
}

and an external receiver $(\mathrm{Rx})$ is currently restricted by low radiation efficiency $\eta \lesssim 0.1 \%$ [11]. Considering typical maximum input power levels ranging from a few to about $50 \mathrm{~mW}$ [1] (limited by safety standards) and Rx sensitivities, this efficiency provides an operating range only up to a few meters.

Poon et al. [12] showed that the optimal wireless powering of an in-body device by an on-body current loop can be achieved at $\mathrm{GHz}$ frequencies (mid-field region). A number of studies suggested that the optimal conditions exist as well for the far-field energy transfer from a body to surrounding free space [13]. Considering the dispersive properties of body tissues as well as attenuation and reflection losses, the optimal conditions should be sought within the $10^{7} \mathrm{~Hz}$ to a few $\mathrm{GHz}$ range. Radiation efficiency within this range depends on geometrical and EM properties of the surrounding tissues, type and depth of the source, its size, polarization, and bandwidth.

To derive the optimal radiation conditions, we start by modeling biological media as an infinite homogeneous half-space with dispersive muscle-equivalent EM properties. A planar interface bounds the half-space with air. Then, we stratify the half-space with skin and fat. Next, we study a stratified cylindrical domain. Finally, we analyze a realistic model of the human abdominal region. We establish the optimal operating frequencies for deep-body (the depth $d \gtrsim 3 \mathrm{~cm}$ ) and for subcutaneous applications $(d \lesssim 3 \mathrm{~cm})$. We also study the surface wave interference and polarization effects on the far-field EM energy transfer efficiency by exciting $\mathrm{TM}_{z}$ (s-polarization) and $\mathrm{TE}_{z}$ modes ( $p$-polarization) through infinitesimal sources: a magnetic dipole and magnetic current, respectively. 

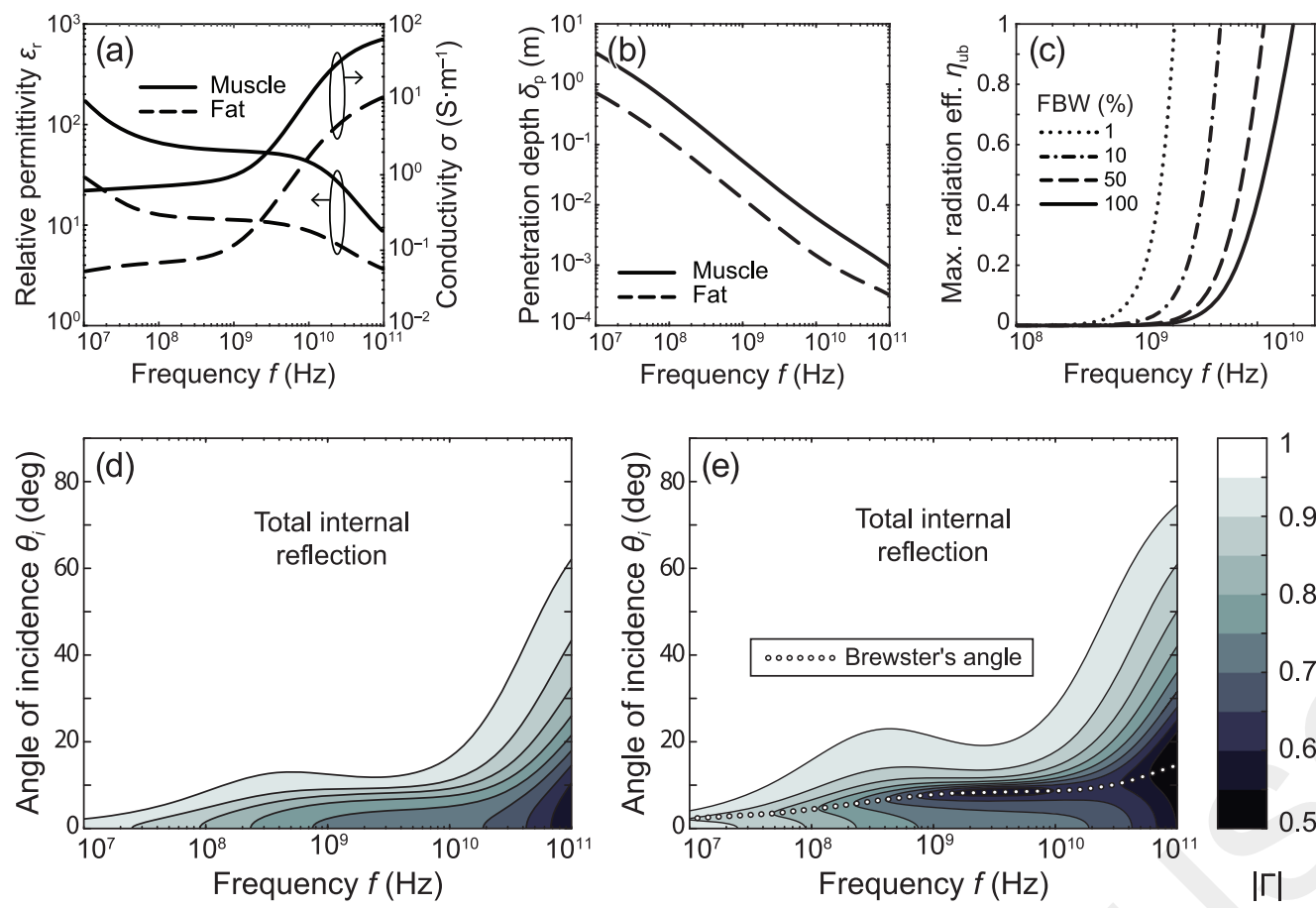

FIG. 1. Mechanisms affecting the radiation efficiency $\eta$ of body-implanted devices. (a) Electromagnetic dispersion of tissues considered in this study (illustrated by EM properties of muscle and fat) obtained using fourregion Cole-Cole model by Gabriel et al. [8-10]. (b) Penetration depths $\delta_{p}=1 / \alpha$ of a plane wave propagating into dispersive muscle and fat tissues. (c) Maximum achievable radiation efficiencies $\eta_{\text {ub }}$ of an antenna in a 0.8 lossless dielectric according to Eq. (3) for given fractional 0.7 bandwidths $\mathrm{FBW}_{2}=f_{0} / \mathrm{BW}$ considering $\mathrm{VSWR}=2$, $0.6 a=5 \mathrm{~mm}$, and the dispersive permittivity of muscle 0.5 tissue. (d)-(e) Reflection coefficients $|\Gamma|$ of a plane wave incident upon an infinite planar dispersive-skin-to-air interface for (d) $s$-polarization and (e) $p$-polarization.

\section{MECHANISMS AFFECTING THE RADIATION EFFICIENCY}

From a generalized EM point of view, body tissues are an anisotropic, nonlinear, heterogeneous, and dispersive medium [14]. Considering radio communication frequencies and related power levels, we will assume here all EM properties as isotropic (permittivity $\hat{\varepsilon}$ is scalar), non-magnetic $\left(\mu=\mu_{0}\right)$, and linear [no heating of tissues due to low supplied power $\left.P_{s}: \Delta T \approx 0 \Rightarrow \hat{\varepsilon} \neq f(\mathbf{E})\right]$. The EM spectrum of the tissue is characterized by three main relaxation regions accounting for $(\boldsymbol{\alpha})$ ionic diffusion and membrane-related mechanisms, $(\boldsymbol{\beta})$ capacitive charging of cellular membranes and intercellular bodies, and $(\gamma)$ dipolar polarization of free water in tissues. Another fourth minor dispersion $(\boldsymbol{\delta})$ can also appear in some protein solutions [15]; the mechanisms causing $\boldsymbol{\delta}$-dispersion are the least studied.

These relaxation regions define the dispersive EM properties of tissues $\hat{\varepsilon}(\omega)=\varepsilon^{\prime}(\omega)-i \varepsilon^{\prime \prime}(\omega)=\varepsilon^{\prime}(\omega)-$ $i \sigma(\omega) /\left(\omega \varepsilon_{0}\right)$, where $\varepsilon^{\prime}=\varepsilon_{r}(\mathbf{r}, \omega) \varepsilon_{0}$ is the permittivity $\left(\mathrm{F} \cdot \mathrm{m}^{-1}\right), \sigma(\mathbf{r}, \omega)$ is the electrical conductivity $\left(\mathrm{S} \cdot \mathrm{m}^{-1}\right), \mathbf{r}$ is the position vector, $\omega=2 \pi f$ is the angular frequency $\left(\mathrm{rad} \cdot \mathrm{s}^{-1}\right)$, and $f$ is the frequency $(\mathrm{Hz})$. Cole-Cole dispersion accurately models the complex permittivity of tissues [16]. In this study, we use the four-region $(\boldsymbol{\alpha}, \boldsymbol{\beta}, \boldsymbol{\gamma}$, and $\boldsymbol{\delta}$ ) Cole-Cole approximation based on the experimental data obtained by Gabriel et al. [8-10]. Figure 1(a) shows the EM properties of muscle and fat according to the model. The $\gamma$-dispersion affects the EM properties from about $10^{9} \mathrm{~Hz}$. For the majority of tissues, relative permittivity $\varepsilon_{r}(\omega) \propto 1 / \omega$, and $\sigma(\omega) \propto \omega$.

Several frequency-dependent mechanisms affect the EM energy transfer efficiency from dispersive body tissues to free space. The major are 1) the attenuation due to dielectric and conductive losses, 2) reflection (mismatch) losses due to impedance contrast, and 3) the physical limitations on the radiation efficiency $\eta$ of electrically small sources in lossy media. Furthermore, a body, its cavities and organs may support resonance modes affecting the radiation efficiency [17].

\section{A. Attenuation in Dispersive Tissue}

For a TEM wave propagating in lossy media, the attenuation constant $\alpha\left(\mathrm{Np} \cdot \mathrm{m}^{-1}\right)$ can be expressed as [20]

$$
\alpha=\omega \sqrt{\mu_{0} \varepsilon(\omega)} \sqrt{\frac{1}{2}\left[\sqrt{1+\left(\frac{\sigma(\omega)}{\omega \varepsilon(\omega)}\right)^{2}}-1\right]} .
$$

Characterizing the propagation loss using the penetration depth $\delta_{p}=1 / \alpha$ gives the depth $(\mathrm{m})$ at which the amplitude of the fields falls by $1 / e$ (about $37 \%$ ) or the intensity by $1 / e^{2}(14 \%)$. Figure $1(\mathrm{~b})$ shows the penetration depth $\delta_{p}$ in dispersive muscle and fat tissues. This mechanism prominently favors lower frequencies to maximize the transmission efficiency. 

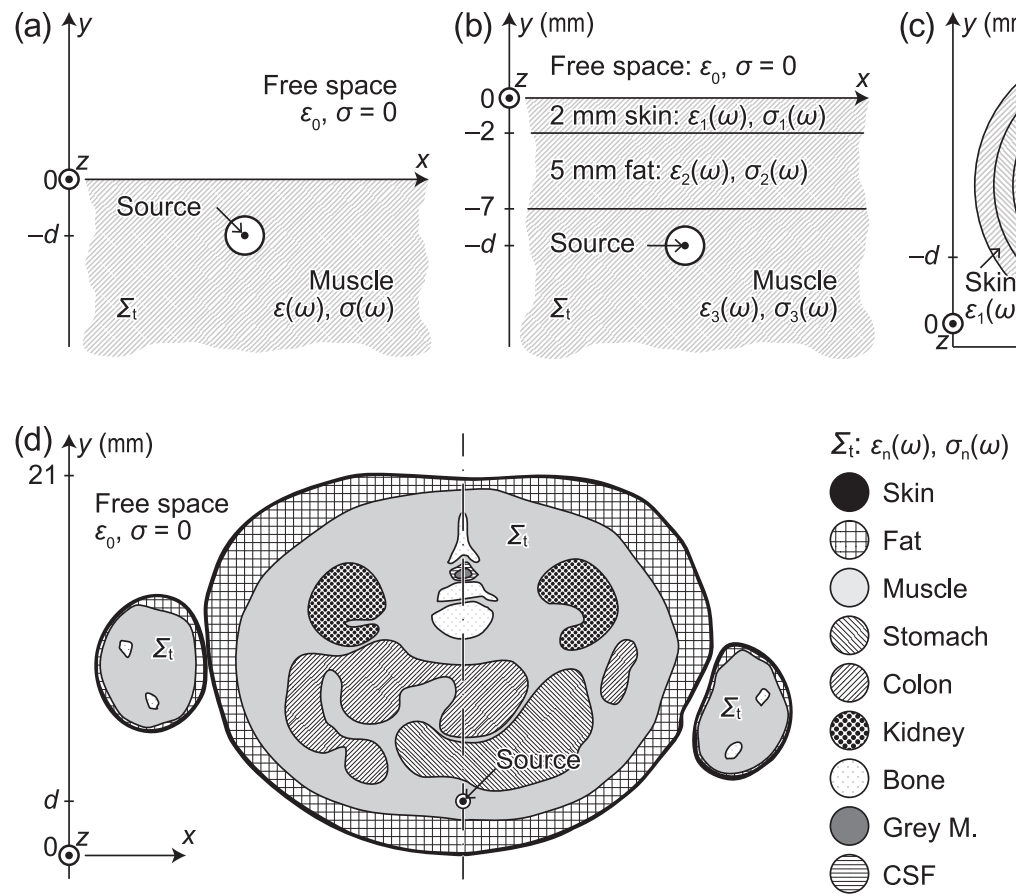
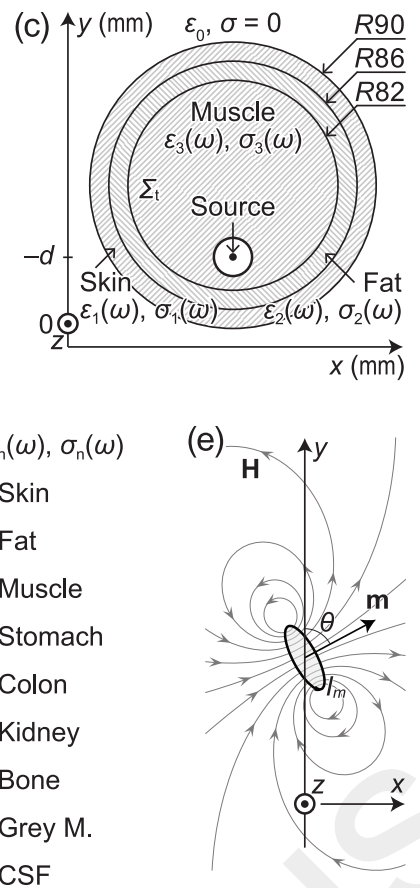

FIG. 2. 2D model formulations used in the study (source regions not to scale, $z$-axis is out of plane). EM properties are according to Gabriel et al. [15]. (a) A planar interface between two semi-infinite homogeneous half-spaces. $\Sigma_{\mathrm{t}}(d<0)$ has dispersive muscle-equivalent $\mathrm{EM}$ properties. (b) A planar stratified heterogeneous model as proposed by Poon et al. [12]. $\quad \Sigma_{\mathrm{t}}(d<0)$ consists of muscle, fat, and skinequivalent layers. (c) Circular heterogeneous phantom (not to scale) similar to the one used in $[18,19] . \Sigma_{\mathrm{t}}(R<90)$ includes dispersive layers with muscle-, fat, and skin-equivalent EM properties. (d) Realistic model of a human abdominal region (nine dispersive tissues). (e) Magnetic dipole of a moment $\mathbf{m}$.

\section{B. Wave Impedance Contrast and Reflection Losses}

As abrupt transitions of EM properties occur on boundaries between tissues [21], the wave impedance mismatch affects the through-body transmission via reflection losses. High contrast arises, for instance, on skin-air and muscle-fat interfaces. Reflection coefficient $\Gamma=E^{-} / E^{+}$, where $E^{-}$is the amplitude of the incident and $E^{+}$ of reflected electric field, depends on wave impedances of two media $Z_{n}=\left[\left(i \omega \mu_{0}\right) /(\sigma+i \omega \varepsilon)\right]^{-1 / 2}, n=$ 1,2 , the angles of incidence $\theta_{i}$ and of refraction $\theta_{t}=$ $\sin ^{-1}\left[\sin \left(\theta_{i}\right) Z_{2} / Z_{1}\right]$, and polarization.

As body-implanted devices radiate mainly from more dense to less dense media (though, the opposite occurs, for instance, on fat-skin and bone-muscle interfaces), a significant part of energy is trapped inside of a body due to the total internal reflection. For the $s$ - (perpendicular) and $p$-polarized (parallel) waves, assuming the plane wave $E(z)=E_{0}^{+} e^{-i \gamma z}+E_{0}^{-} e^{+i \gamma z}$ solution of (4), where $\gamma$ is the complex propagation constant [20]:

$$
\begin{aligned}
& \Gamma_{s}=\left(Z_{2} \cos \theta_{i}-Z_{1} \cos \theta_{t}\right) /\left(Z_{2} \cos \theta_{i}+Z_{1} \cos \theta_{t}\right), \\
& \Gamma_{p}=\left(-Z_{1} \cos \theta_{i}-Z_{2} \cos \theta_{t}\right) /\left(Z_{1} \cos \theta_{i}+Z_{2} \cos \theta_{t}\right) .
\end{aligned}
$$

Figures 1(d) and 1(e) show the angular decomposition of (2) for a plane wave incident upon a dispersive-skin-air interface. For $\theta_{i}>0, p$-polarization gives better transmission with $\max (|\Gamma|=0.5)$ at Brewster's angle. Opposing the attenuation in tissues, the reflection losses favor using higher frequencies. Considering that the relative role of attenuation in the total loss increases with the depth of a source in lossy tissues, the optimal operating frequency would be inversely proportional to the depth.

\section{Fundamental Limitations on Radiation Efficiency of Electrically Small Sources}

This mechanism is intrinsic to the source rather than to the medium properties. By definition [22], a source is electrically small (ESS) when $k a<0.5$, where $k=2 \pi / \lambda$ is the wavenumber $\left(\mathrm{m}^{-1}\right), \lambda(\varepsilon)$ is the wavelength $(\mathrm{m})$, and $a$ is the radius of the source circumsphere. Physical limitations of ESS in terms of the minimum quality factor $Q_{\mathrm{LB}}=\eta\left[(k a)^{-3}+(k a)^{-1}\right][23,24]$ are valid for the source in a lossless medium. To apply it for the one radiating into lossy media, two assumptions must be made: (1) the currents induced in lossy tissues reradiate negligible energy, and (2) assuming the Thevenin's equivalent circuit [25], the loss resistance $R_{L}$ accounts for the dissipation in tissue. This approximation is too crude for an accurate evaluation of the lower bound on $Q_{\mathrm{LB}}$ (it improves with lower conductivity $\sigma)$. However, it shows how the maximum achievable efficiency $\eta_{\mathrm{ub}}$ relates to $Q_{\mathrm{LB}}$ and $k a[26] . Q$ can be expressed in terms of the fractional bandwidth of the source (FBW) at a given voltage standing wave ratio (VSWR) level as $Q=\mathrm{FBW}(\mathrm{VSWR}-1) / \sqrt{\mathrm{VSWR}}[27]$. In this way, the approximate maximum achievable radiation efficiency $\eta_{\mathrm{ub}}$ in a lossless dielectric for a given FBW is

$$
\eta_{\mathrm{ub}}=\mathrm{FBW} \frac{\mathrm{VSWR}-1}{\sqrt{\mathrm{VSWR}}} \frac{[k(\varepsilon) a]^{3}}{[k(\varepsilon) a]^{2}+1} .
$$

Eq. (3) implies that radiation efficiency must be sacrificed to achieve the bandwidth over the ESS limit [Figure 1(c)]. Using higher frequencies allows one to maximize the $\eta_{\mathrm{ub}} / \mathrm{FBW}$ ratio. 


\section{PROBLEM FORMULATION}

The aforementioned mechanisms define the achievable radiation efficiency of body-implanted devices. The efficiency depends on the frequency, source position and depth in a body, arrangement of surrounding tissues, and polarization.

The source forms a coupled system of accelerating charges with the tissues. This system radiates to surrounding free space due to non-conserved current and charge in the localized region surrounding the source [28]. The inhomogeneous wave equation models the behavior of EM field from an arbitrary source. In terms of the time-harmonic (time variations of the form $e^{i \omega t}$ ) electric field $\mathbf{E}\left(\mathrm{V} \cdot \mathrm{m}^{-1}\right)$, it is expressed as [20]

$$
\begin{aligned}
\nabla^{2} \mathbf{E}= & \nabla \times \mathbf{M}_{i}+i \omega \mu_{0} \mathbf{J}_{i}+\frac{1}{\varepsilon} \nabla q_{e v} \\
& +i \omega \mu_{0} \sigma \mathbf{E}-\omega^{2} \mu_{0} \varepsilon \mathbf{E},
\end{aligned}
$$

where $\mathbf{M}_{i}$ is the source magnetic current density $\left(\mathrm{V} \cdot \mathrm{m}^{-2}\right), \quad \mathbf{J}_{i}$ is the source electric current density $\left(\mathrm{A} \cdot \mathrm{m}^{-2}\right)$, and $q_{e v}$ is the electric charge density $\left(\mathrm{C} \cdot \mathrm{m}^{-3}\right)$. Assuming $\mathbf{E}(x, y, z)=\mathbf{E}(x, y) e^{i k_{z} z}$ where $k_{z}$ is the outof-plane wavenumber, we reduce the spatial dimensionality of the problem.

From the conservation-of-energy point of view (Poynting's theorem),

$$
\begin{array}{r}
\overbrace{-\frac{1}{2}\left(\mathbf{H}^{*} \cdot \mathbf{M}_{i}+\mathbf{E} \cdot \mathbf{J}_{i}^{*}\right)}^{P_{s}}=\overbrace{\nabla \cdot\left(\frac{1}{2} \mathbf{E} \times \mathbf{H}^{*}\right)}^{P_{e}} \\
+\underbrace{\frac{1}{2} \sigma|\mathbf{E}|^{2}}_{P_{d}}+\underbrace{i 2 \omega\left(\frac{1}{4} \mu_{0}|\mathbf{H}|^{2}-\frac{1}{4} \varepsilon|\mathbf{E}|^{2}\right)}_{i 2 \omega\left(W_{m}-W_{e}\right)}
\end{array}
$$

where $\mathbf{H}$ is the magnetic field $\left(\mathrm{A} \cdot \mathrm{m}^{-1}\right), P_{s}$ is the supplied power $(\mathrm{W}), P_{e}$ is the exiting power $(\mathrm{W}), P_{d}$ is the dissipated power, and $W_{m}, W_{e}$ are the time-average magnetic and electric energies, respectively.

Plane-wave solutions of (4) given in Section II show how each mechanism affects the radiation efficiency. However, to accurately evaluate the relative importance of each mechanism given a particular depth, one needs a numerical solution of (4). Annex A outlines the numerical approach used in this study.

We formulate the problem geometry increasing its complexity from a simple to anatomically realistic one (Figure 2). First, we consider a planar homogeneous medium consisting of two infinite half-spaces that represent a free space domain and lossy dispersive tissue domain $\Sigma_{t}$ [Figure 2(a)]. Then, we study a planar layered medium by adding $2 \mathrm{~mm}$ skin and $5 \mathrm{~mm}$ fat layers to the $\Sigma_{\mathrm{t}}$ [as proposed in [12], Figure 2(b)]. Next, we model the human body as a heterogeneous circular stratified domain $\Sigma_{\mathrm{t}}$ with the dimensions used in [18] [Figure 2(c)]. Finally, we analyze the heterogeneous realistic model of a human abdominal region [Figure $2(\mathrm{~d})$ ].
We consider magnetic sources as they give better efficiency in lossy dielectrics due to non-magnetic properties of biological tissues $\left(\mu \approx \mu_{0}\right)[12,19]$. Two sources have been studied: 1) a magnetic dipole of a moment $\mathbf{m}$ oriented at angles $\theta=0^{\circ}, 45^{\circ}, 60^{\circ}$, and $90^{\circ}$ [Figure 2(e)] that excite $\mathrm{TM}_{z}$ mode of the first angular order (by convention, $\mathbf{m}$ aligns with the the $y$-axis at $0^{\circ}$ and $x$-axis at $\left.90^{\circ}\right) ; 2$ ) out-of-plane magnetic current $I_{m}$ that excite $\mathrm{TE}_{z}$ mode of zeroth angular order.

The elementary source is centered within a lossless $\left(\sigma=0 \mathrm{~S} \cdot \mathrm{m}^{-1}\right) \varnothing 4 \mathrm{~mm}$ area bounded by a closed contour $C_{S}$ on which the supplied specific power $P_{s}\left(\mathrm{~W} \cdot \mathrm{m}^{-1}\right)$ can be evaluated independently of the source formulation as

$$
P_{s}=\oint_{C_{S}}\left(\frac{1}{2} \mathbf{E} \times \mathbf{H}^{*}\right) \cdot \mathrm{d} \mathbf{s} .
$$

The exiting specific power $P_{e}\left(\mathrm{~W} \cdot \mathrm{m}^{-1}\right)$ is evaluated on the contour $C_{\mathrm{FF}}$ (located at the distance from the source satisfying the far-field criterion) as

$$
P_{e}=\int_{C_{\mathrm{FF}}}\left(\frac{1}{2} \mathbf{E} \times \mathbf{H}^{*}\right) \cdot \mathrm{d} \mathbf{s} .
$$

To verify the conservation-of-energy condition (5), the dissipated specific power $P_{d}\left(\mathrm{~W} \cdot \mathrm{m}^{-1}\right)$ is evaluated over all lossy domains $\Sigma_{\mathrm{t}}$ as

$$
P_{d}=\frac{1}{2} \iint_{\Sigma_{\mathrm{t}}} \sigma(\mathbf{r}, \omega)|\mathbf{E}|^{2} \mathrm{~d} x \mathrm{~d} y .
$$

The radiation efficiency of an ideal source is then

$$
\eta_{\text {idl }}(\omega) \equiv P_{e} / P_{s} \text {. }
$$

Taking into account the maximum radiation efficiency limitations (3) of an ESA for a given BW and assuming the dissipation in tissues as an equivalent loss resistance of the antenna $R_{L}$ (see Section II C):

$$
\eta_{\mathrm{rad}}(\omega) \equiv \min \left[\eta_{\mathrm{idl}}, \eta_{\mathrm{ub}}\right] \text {. }
$$

For a realistic antenna, the total efficiency estimate also includes the mismatch loss of the antenna as $\eta_{\text {tot }}=$ $\eta_{\text {rad }}\left(1-\left|\Gamma_{\mathrm{A}}\right|^{2}\right)$, where $\Gamma$ is the reflection coefficient at the input terminals of the antenna. In this study, we consider perfectly matched sources $\left(\Gamma_{\mathrm{A}}=0\right)$.

To compare $\mathbf{E}$-field distribution surface plots for given $f, d$, and source formulations, we normalize $\|\mathbf{E}\|$ by the supplied power $P_{s}(6)$ and the intrinsic impedance of the media $Z_{n}$ surrounding the source as $\log _{10}\left(\|\mathbf{E}\| / \sqrt{Z P_{s}}\right)$.

Finally, to quantify the reflection losses, we study attenuation in the planar homogeneous medium [Figure 2(a)] by removing reflection at the interface between two half-spaces. We accomplish it by equaling the wave impedance $Z_{n}$ of the top half-space to the muscleequivalent bottom one $\Sigma_{\mathrm{t}}(d<0)$. It enables evaluating the absolute and relative roles of reflection as

$$
\begin{aligned}
\Delta \eta(\omega) & \equiv \eta_{\mathrm{att}}-\eta_{\mathrm{idl}}, \\
\delta \eta(\omega) & \equiv 1-\eta_{\mathrm{idl}} / \eta_{\mathrm{att}},
\end{aligned}
$$

where $\eta_{\text {att }}=1-P_{d} / P_{s}$ is the efficiency without reflection. 

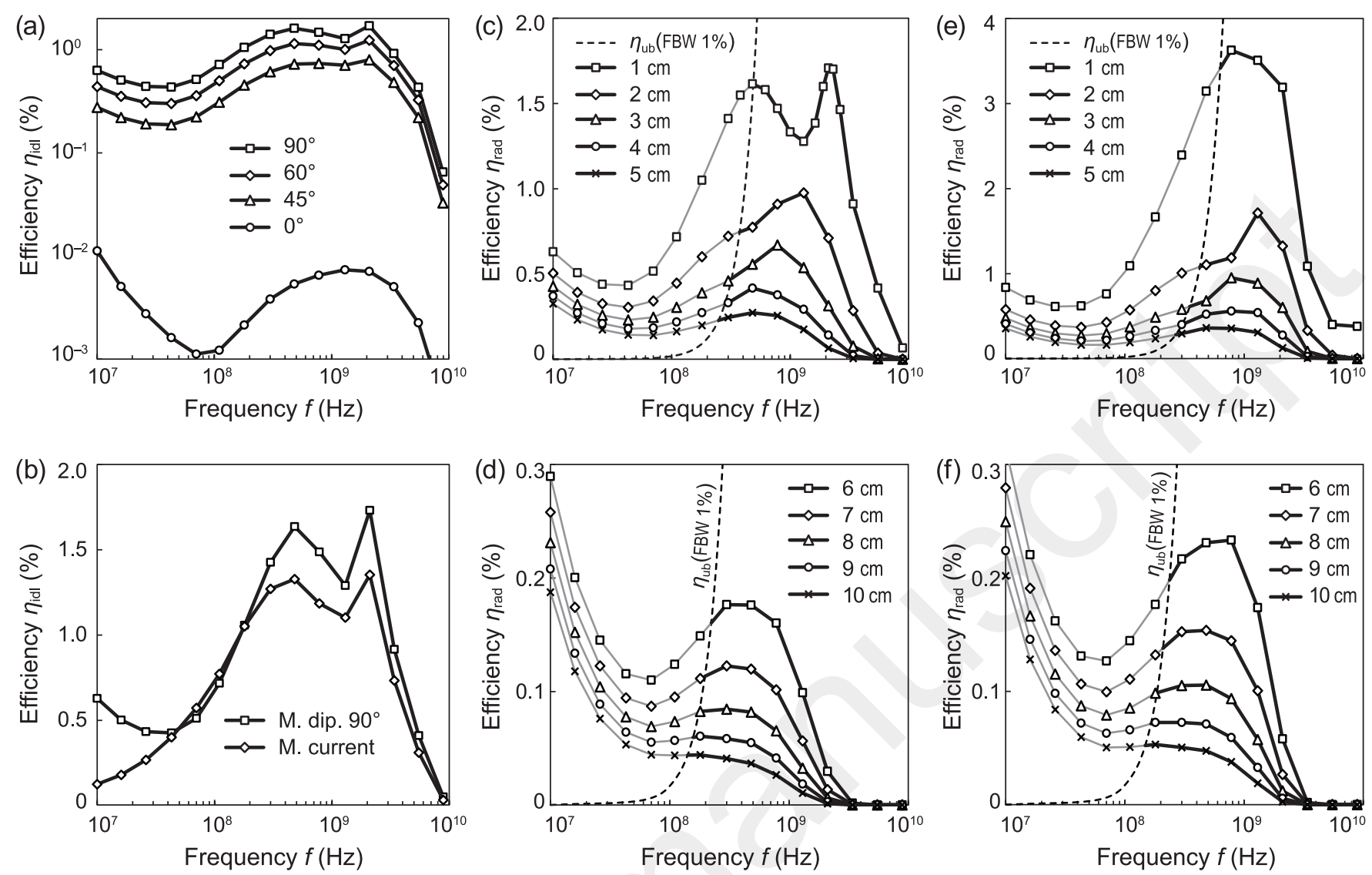

FIG. 3. Radiation efficiencies $\eta_{\text {idl }}$ and $\eta_{\text {rad }}$ in infinite planar-bounded media. (a) Orientation of the magnetic dipole moment m strongly affects the efficiency (homogeneous half-space). (b) The $90^{\circ}$-oriented magnetic dipole moment $\mathbf{m}$ ( $\mathrm{TM}_{z}$ mode) compared to the out-of-plane magnetic current source $\left(\mathrm{TE}_{z}\right.$ mode) at $d=1 \mathrm{~cm}$ in the homogeneous half-space medium. (c) The homogeneous half-space medium at $d=1$ to $5 \mathrm{~cm}$ and (d) at $d=6$ to $10 \mathrm{~cm}$ implantation depths. (e) The layered (skin-fat-muscle) half-space medium at $d=1$ to $5 \mathrm{~cm}$ and (f) at $d=6$ to $10 \mathrm{~cm}$ implantation depths.
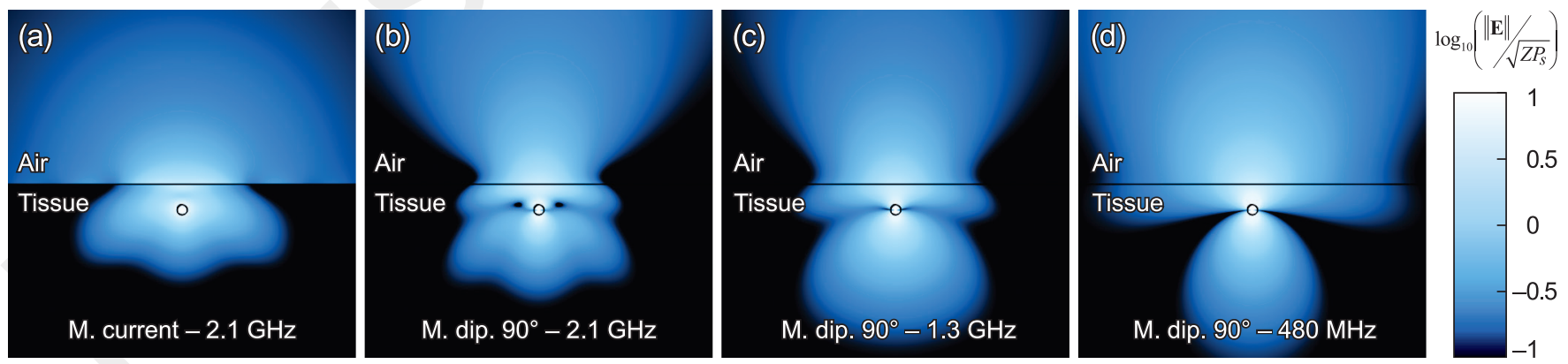

FIG. 4. Normalized E-field distributions at $d=1 \mathrm{~cm}$ in infinite planar-bounded homogeneous half-space media. (a) The magnetic current source $\left(\mathrm{TE}_{z}\right.$ mode) and (b)-(c)-(d) The $90^{\circ}$-oriented magnetic dipole $\left(\mathrm{TM}_{z}\right.$ mode). The radiation efficiency peaks at $2.1 \mathrm{GHz}(\mathrm{a})-(\mathrm{b})$ and $480 \mathrm{MHz}$ (d) for both sources and drops at $1.3 \mathrm{GHz}$ (c) due to the interference with reflected wave. 


\section{RESULTS IN PLANAR-BOUNDED INFINITE MEDIA}

\section{A. Homogeneous Half-Space}

We start with an arbitrary-oriented magnetic dipole in an infinite homogeneous planar-bounded half-space with muscle-equivalent EM properties [Figure 1(a)]. Figure $3(\mathrm{a})$ shows the efficiencies $\eta_{\text {idl }}$ for four dipole moment $\mathbf{m}$ orientations $\left(\theta=0^{\circ}, 45^{\circ}, 60^{\circ}\right.$, and $\left.90^{\circ}\right)$ at $d=1 \mathrm{~cm}$. The efficiency strongly depends on $\theta$ and peaks about $2 \mathrm{GHz}$ for all orientations. The extremely low efficiency at $\theta=0^{\circ}$ is due to the fact that both radiation lobes of the dipole are pointing towards the direction parallel to the infinite planar interface. The optimal frequency is invariant to the orientation of the magnetic dipole moment $\mathbf{m}$. Therefore, for further studies we proceed with the $\theta=90^{\circ}$ alignment that gives the highest efficiency $\eta_{\text {idl }}$ and thus minimizes the computational error. For the magnetic current source, the reduced overall efficiency is due to the radiation isotropy [Figure 4(a)] in comparison to the first-angular-order $\mathrm{TM}_{z}$ mode of the magnetic dipole source [Figure 4(b)]. The optimal frequency, however, remains invariant to the source formulation [Figure 3(b)].

Analysis in the homogeneous half-space medium [Figure $3(\mathrm{c})$ and $3(\mathrm{~d})$ ] reveals that the optimal frequency range is inversely proportional to the depth $d$ of the source and decays exponentially (Figure 5). At $d=1 \mathrm{~cm}$ $\left[\eta_{\text {idl }}(f)\right.$ on Figure $\left.3(\mathrm{c})\right]$, the radiation efficiency peaks at $480 \mathrm{MHz}$ and $2.1 \mathrm{GHz}$ but drops in-between at $1.3 \mathrm{GHz}$. At the global maximum $[2.1 \mathrm{GHz}$, Figure $4(\mathrm{a})$ and $4(\mathrm{~b})]$ ), the destructive interference occurs close to the $\Sigma_{\mathrm{t}}$ interface reducing the area of dissipated power independently of the source formulation. On the other hand, at the local minimum $[1.3 \mathrm{GHz}$, Figure $4(\mathrm{c})]$, one can notice the enlarged back lobe [compared to the local maximum at $480 \mathrm{MHz}$, Figure 4(d)] as well as increased power absorption around the zeros of the dipole radiation pattern (collinear with the the moment $\mathbf{m}$ ). As the position of the local minimum is invariant to the source formulation and the orientation of $\mathbf{m}$ (except for $\theta=0^{\circ}$ where the source does not radiate in the direction of the interface), the effect is due to the $f / d$ relation and consequently to interference with the standing surface wave appearing at the $\Sigma_{\mathrm{t}}$ interface. Christ et al. [29] reported a similar effect: reflection at the fat-muscle interface excites a standing wave in subcutaneous fat increasing the specific absorption rate (SAR) by about $3 \mathrm{~dB}$.

For the deep-implanted magnetic dipole $(d=6-10 \mathrm{~cm})$ [Figure 3(d)], the attenuation losses overtake at higher frequencies, and the peak efficiency $\eta_{\text {idl }}(15)$ drops below $10 \mathrm{MHz}$ (even though local maxima remain in the $2 \times 10^{8}-8 \times 10^{8} \mathrm{~Hz}$ range). However, these efficiencies are unattainable for a reasonable bandwidth of a real source (e.g. $\mathrm{FBW}_{2}>1 \%$ in dielectric). The practically achievable radiation efficiency $\eta_{\text {rad }}(10)$ locates below the $f<1 \mathrm{GHz}$ range for deep implantations (Figure 5).

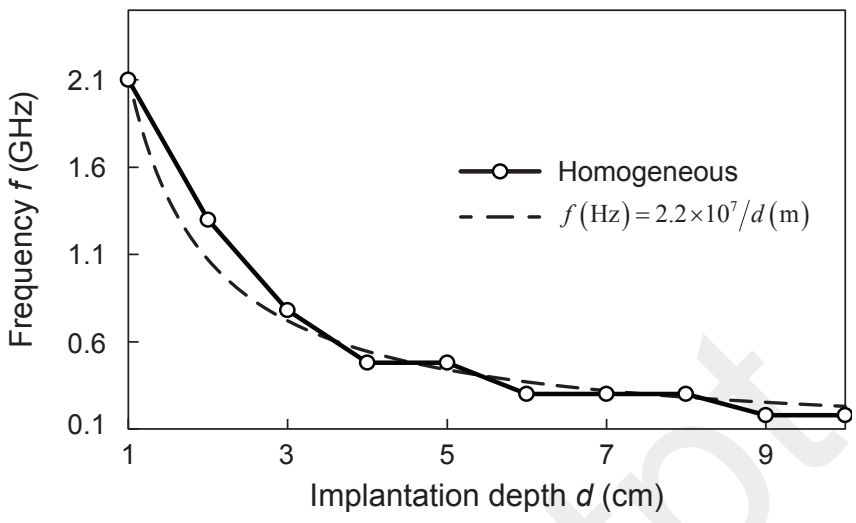

FIG. 5. Optimal frequency $f$ of a source in the infinite planarbounded homogeneous half-space medium (dispersive muscleequivalent EM properties).

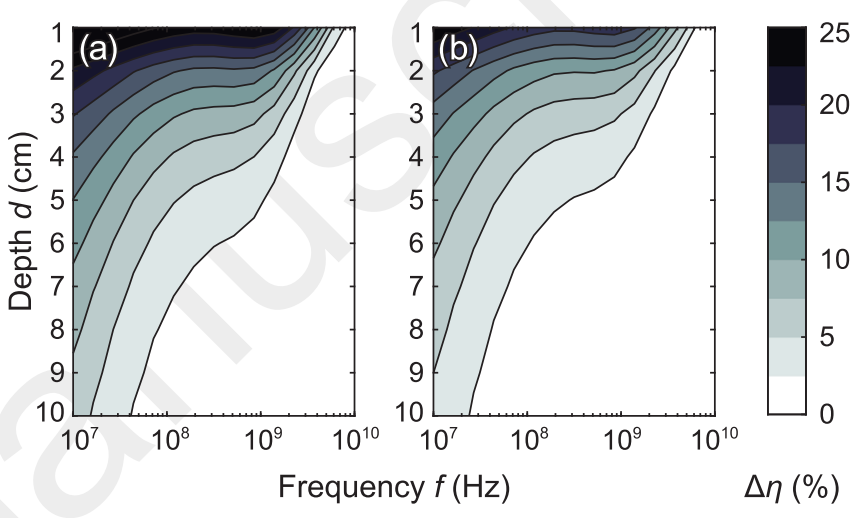

FIG. 6. Absolute reflection losses defined as the efficiency difference $\Delta \eta$ (11a) for (a) magnetic dipole $\left(\mathrm{TM}_{z}\right.$ mode) and (b) magnetic current ( $\mathrm{TE}_{z}$ mode) sources in infinite half-space with muscle-equivalent EM properties.

For the energy transfer from the infinite planarbounded homogeneous half-space with muscle equivalent EM properties to surrounding free space, the maxima of radiation efficiency shifts from about $2 \mathrm{GHz}$ for $d=1 \mathrm{~cm}$ down to below $200 \mathrm{MHz}$ for $d=10 \mathrm{~cm}$ [Figure 5]. This frequency range is about two times lower than the one derived by Poon et al. [12] for the wireless power transfer in mid field from an on-body source region to an implant.

\section{B. Role of Reflection Losses}

Figure 6 shows how much of the radiation efficiency $\eta_{\text {idl }}$ is lost in absolute terms due to reflection losses (11a) at a given implant depth $d$. Within the studied frequency and depth ranges, $80-99 \%$ of the relative efficiency loss $\delta \eta$ (11b) is due to the reflection - not predominantly attenuation as commonly believed [12]. The lower the frequency is, the higher the relative loss. Exploiting $p$-polarization of the $\mathrm{TE}_{z}$ mode [Figure 1(e)] may improve the transmission efficiency by a few percent, and this effect is inversely proportional to the frequency and depth. 

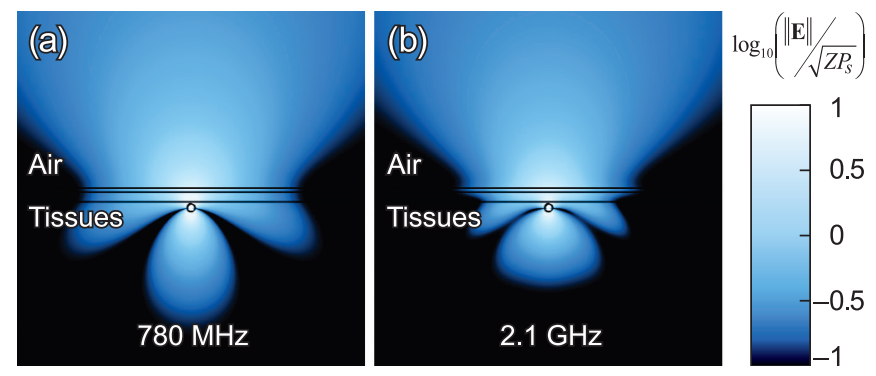

FIG. 7. Normalized E-field distributions at $1 \mathrm{~cm}$ depth in the infinite planar-bounded layered half-space medium. The $90^{\circ}$ oriented magnetic dipole moment $\mathbf{m}\left(\mathrm{TM}_{z}\right.$ mode) at (a) $780 \mathrm{MHz}$ (maximum efficiency) and (b) $2.1 \mathrm{GHz}$.

\section{Stratified Half-Space}

Here, we study the effect of skin and subcutaneous fat layer on the optimal frequency. Figures 3(e) and 3(f) show the evolution of the efficiency peak for 1-10 cm implantation depths. The fat layer acts as a low-loss matching layer, reducing the reflection losses and thus increasing overall efficiency compared to the homogeneous half-space medium [Figures 3(c) and 3(d)] by up to a factor of two. At $1 \mathrm{~cm}$, the peak around $2 \mathrm{GHz}$ disappears shifting the optimal frequency down to below $1 \mathrm{GHz}$. Figure 7(a) shows the normalized E-field distribution at the global maximum of $\eta_{\mathrm{rad}}$ at $780 \mathrm{MHz}$. Destructive interference with the surface wave reduces the width of the dipole back lobe thus minimizing the power absorption at this frequency. On the other hand, at $2.1 \mathrm{GHz}$ [Figure 7(b)] the back lobe is significantly wider. Albeit the destructive interference reduces the front lobe $\mathbf{E}$-field in the fat layer (which is practically lossless), one can notice the higher intensity in the lossy skin (the outer) layer of the half-space $\Sigma_{t}$. The wide back lobe and the amplified intensity in the skin layer together contribute to the increased power dissipation at $2.1 \mathrm{GHz}$ - the global optimum for the homogeneous half-space medium.

A sharp $\eta_{\text {idl }}$ peak appears at about $1.3 \mathrm{GHz}$ for $2 \mathrm{~cm}$ depth [Figure 3(e)] that increases the optimal frequency with the depth (compared to $d=1 \mathrm{~cm}$ ). Again, at this depth the observed resonance behavior plays a significant role, and for the shallow implantation $(d \leq 2 \mathrm{~cm})$ the optimal frequency does not follow the exponential decay on Figure 5 . However, the $f=2.2 \times 10^{7} / d$ approximation is still valid for deeper implantation $(d=2 \mathrm{~cm}$ to $10 \mathrm{~cm})$.

\section{RESULTS IN FINITE-SIZE PHANTOMS}

\section{A. Cylindrical Stratified Phantom}

Next, we model a human body as an infinitelylong three-tissue stratified cylinder (Figure 2c) with the same radii as for the spherical phantom used by Merli et al. [18], Chrissoulidis and Laheurte [19]. Consi-
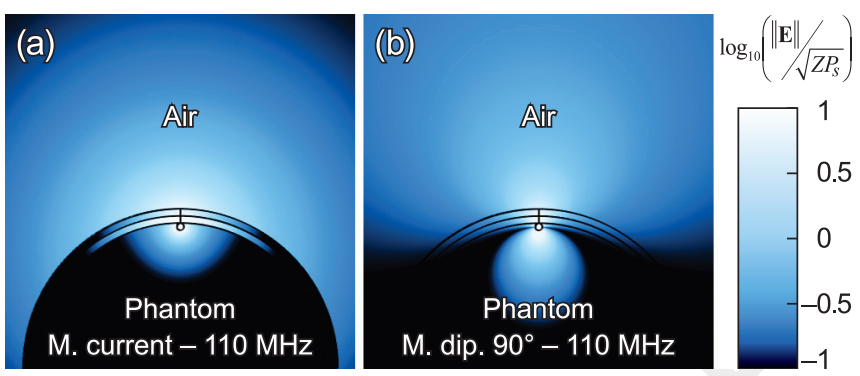

FIG. 8. Normalized E-field distributions at $110 \mathrm{MHz}$ and $1 \mathrm{~cm}$ depth in the cylindrical stratified phantom. (a) The magnetic current source ( $\mathrm{TE}_{z}$ mode). (b) The $90^{\circ}$ oriented magnetic dipole moment $\mathbf{m}\left(\mathrm{TM}_{z}\right.$ mode $)$.

dering the magnetic dipole source $\left(\mathrm{TM}_{z}\right.$ mode $)$ at $d=1-$ $4 \mathrm{~cm}$, the efficiency $\eta_{\text {idl }}$ is about five times higher than the one for the planar media due to 1) weakened attenuation in the finite-sized lossy domain $\Sigma_{\mathrm{t}}$ and 2) reduced effect of the total internal reflection [Figures 1(d) and 1(e)] because of the tissue-air boundary encircling the source (Figure 8).

The efficiency $\eta_{\text {idl }}$ remains on its peak from $20 \mathrm{MHz}$ to $500 \mathrm{MHz}$ for $d=1-3 \mathrm{~cm}$. The magnetic current source [Figure 9(a)] gives results similar to the magnetic dipole in the planar layered medium [cf. Figure 3(e)] with a clear peak near $1 \mathrm{GHz}$. For deeper implantations $\left(d=5_{-}\right.$ $9 \mathrm{~cm}$ ), the efficiency peaks roughly at about $0.5 \mathrm{GHz}$ for both sources [Figures 9(b) and 9(d)].

Two sources behave differently at lower frequencies $\left(f \lesssim 5 \times 10^{8} \mathrm{~Hz}\right)$, especially for the shallow implantation. Here, the efficiency of $\mathrm{TM}_{z}$ remains on its maximum without dropping below $10^{8} \mathrm{~Hz}$. Besides, one can notice the same (though less pronounced) effect on the Figure 3(b) below $3 \times 10^{7} \mathrm{~Hz}$. This may seem surprising as one expects the opposite: $p$-polarized $\mathrm{TE}_{z}$ mode should give better efficiency for the lower frequency range where the reflection losses prevail. Therefore, another mechanism dominates in this case.

As the fat has much lower $\varepsilon_{r}$ and $\sigma$ than muscle [Figure $1(\mathrm{a})], d / \lambda(\omega) \ll 1$ for a shallow source. Considering the finite $\Sigma_{t}$ and its boundaries bending towards the source, the reflection becomes inessential for both modes at the lower frequencies. A quasi-static approach explains this phenomena through the interface boundary conditions for the $\mathbf{E}$-components that are parallel $\left(E_{\|}\right)$and perpendicular $\left(E_{\perp}\right)$ to the air-tissue boundary: $E_{\| a}=E_{\| t}$ and $E_{\perp a}=E_{\perp t}\left(\sigma_{t}+i \omega \varepsilon_{t}\right) /\left(\sigma_{a}+i \omega \varepsilon_{a}\right)$. For $\mathrm{TE}_{z}$ mode, while $\mathbf{E}$ decays along the muscle-fat and skin-air interfaces, it also orients itself along the boundary. In fat and air layers - that have much lower permittivity than the muscle and skin- $\mathbf{E}$ responds by increasing the magnitude of its normal component to satisfy the boundary conditions. Field discontinuities appear at the interfaces [Figure 8(a)]. From $f \lesssim 5 \times 10^{8} \mathrm{~Hz}, \mathbf{E}$ above the phantom $(y>90 \mathrm{~mm})$ opposes the bottom one $(y<90 \mathrm{~mm})$ diminishing the power radiated into the far field. At $f \approx 5 \times 10^{8} \mathrm{~Hz}$, the field re-aligns maximizing 

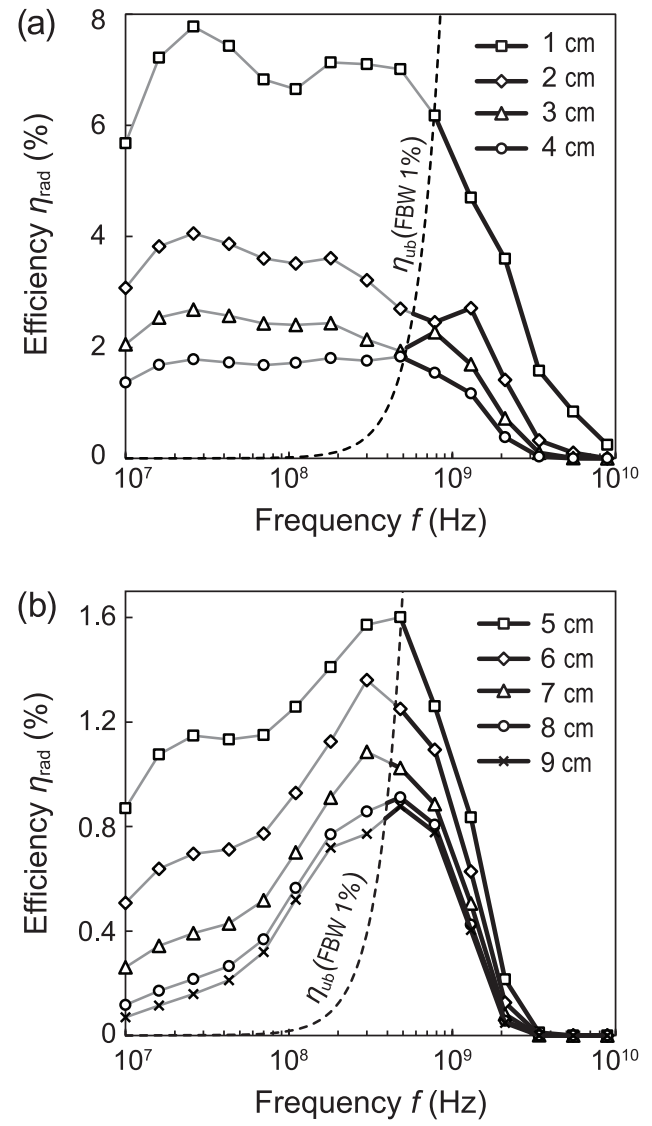
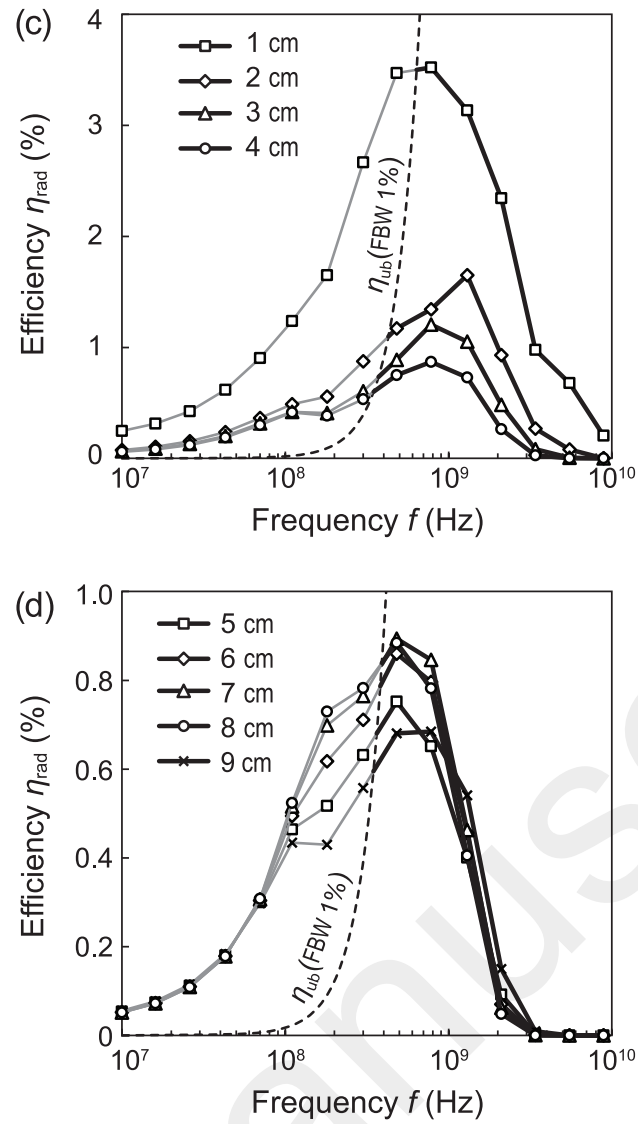

FIG. 9. Radiation efficiencies $\eta_{\text {idl }}$ and $\eta_{\text {rad }}$ for the $R 90 \mathrm{~mm}$ cylindrical stratified phantom similar to the one used by Merli et al. [18], Chrissoulidis and Laheurte [19]: $4 \mathrm{~mm}$ skin-equivalent outer layer, $4 \mathrm{~mm}$ fat-equivalent intermediate layer, and $R 82$ muscle-equivalent domain containing the source. Dispersive tissue EM properties of the layers are according to Gabriel et al. [15]. (a) The $90^{\circ}$ oriented magnetic dipole moment $\mathbf{m}\left(\mathrm{TM}_{z}\right.$ mode $)$ source at $d=1$ to $4 \mathrm{~cm}$ and (b) $d=5$ to $9 \mathrm{~cm}$ implantation depths. (c) The out-of-plane magnetic current source $\left(\mathrm{TE}_{z}\right.$ mode) at $d=1$ to $4 \mathrm{~cm}$ and (d) $d=5$ to $9 \mathrm{~cm}$ implantation depths. At $d=9 \mathrm{~cm}$, the source is in the middle of the phantom. $\eta_{\text {idl }}$ [Figure 9(c)].

For the $\mathrm{TM}_{z}$ mode, $\mathbf{E}$ is continuous through the interfaces [Figure 8(b)]. The field at the bottom of the phantom is thus negligible and does not diminish the radiation efficiency at lower frequencies (Figure 9(a)). As the source goes deeper into the phantom $[d / \lambda(\omega)$ increases], reflection losses kick in again reducing the efficiency [Figure 9(b)] as within the infinite media.

As a final remark, even though $\mathrm{TM}_{z}$ mode gives high $\eta_{\text {idl }}$ at $f<10^{8} \mathrm{~Hz}$ for subcutaneous applications near curved boundaries, these efficiencies are practically unachievable for a reasonable bandwidth $\left(\mathrm{FBW}_{2}>1 \%\right.$ in dielectric with muscle-equivalent permittivity).

\section{B. Anatomical Torso Phantom}

Finally, with the understanding built on how reflection and attenuation losses depend on source formulation and affect propagation in the canonical models, we proceed to the anatomical (abdominal) phantom [Figure 2(d)]. The implantation depth varies along the $y$-axis from $1 \mathrm{~cm}$ to $20 \mathrm{~cm}$. At $20 \mathrm{~cm}$, the source is again about $1 \mathrm{~cm}$ far from the skin-air interface.

Figure 10 shows the radiation efficiencies for both source formulations. The optimal frequency range for deep implantation $(d \gtrsim 2 \mathrm{~cm})$ is congruent with the canonical models (Figure 5). As for the cylindrical stratified phantom, the same effect shows up at the $f<10^{8} \mathrm{~Hz}$ range, of the increased efficiency of $\mathrm{TM}_{z}$ mode compared to $\mathrm{TE}_{z}$. The $\mathbf{E}$-field distributions for both sources at $10^{8} \mathrm{~Hz}$ are given in Figure 11(a) and 11(e). For the magnetic current source, a large part of total $\mathbf{E}$ is contained in fat layer. Within the optimal frequency range, $\mathrm{TM}_{z}$ mode gives about $20 \%$ higher efficiency than $\mathrm{TE}_{z}$ mode for all implantation depths due both to higher directivity and lower reflection losses.

At $1 \mathrm{~cm}$ depth, the efficiency drops at about $2.1 \mathrm{GHz}$ within the optimal range independently of the excitation mode as for the planar homogeneous model [Figure 10(a) and 10(d); E distributions on Figure 11(c) and 11(g)]. The global efficiency maximum appears around $800 \mathrm{MHz}$ [Figure 11(b) and (f)] and a local one at $3.4 \mathrm{GHz}[\mathrm{Fi}-$ gure $11(\mathrm{~d})$ and $11(\mathrm{~h})]$. The local minimum is at $2.1 \mathrm{GHz}$. The efficiency $\eta_{\text {idl }}$ at $800 \mathrm{MHz}$ exceeds the one observed at $2.1 \mathrm{GHz}$ by about 6.5 times. As for the planar homogeneous model, the drop of the efficiency is due to constructive interference with the standing surface wave that increases power absorption in tissue.

For the deep-body implantation [more than $2 \mathrm{~cm}$ from the skin, Figure 10(b) and 10(e)], the radiation efficiency optimum spreads within $10^{8}-10^{9} \mathrm{~Hz}$ range. Figure 12 shows the E-field distribution for magnetic dipole and current sources at $6 \mathrm{~cm}$ depth. The maximum $\mathbf{E}$ outside the phantom localizes where the attenuation path is shorter and contains less interfaces between tissues. Pola- 

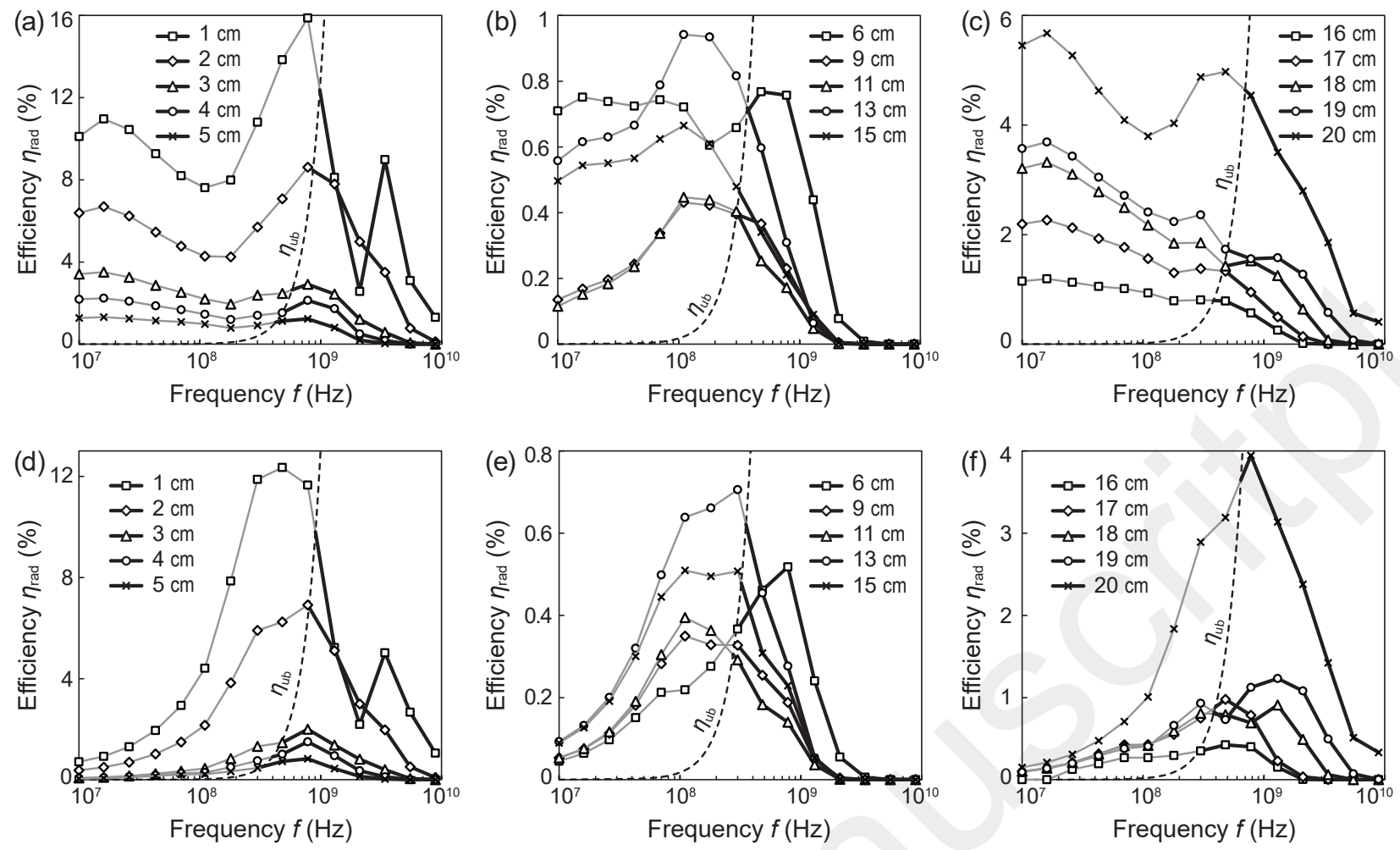

FIG. 10. Radiation efficiencies $\eta_{\mathrm{rad}}$ in the anatomical phantom at $d=1$ to $20 \mathrm{~cm}$ implantation depths. (a)-(c) The $90^{\circ}$ oriented magnetic dipole moment $\mathbf{m}\left(\mathrm{TM}_{z}\right.$ mode). (d)-(f) The out-of-plane magnetic current source (TE mode).
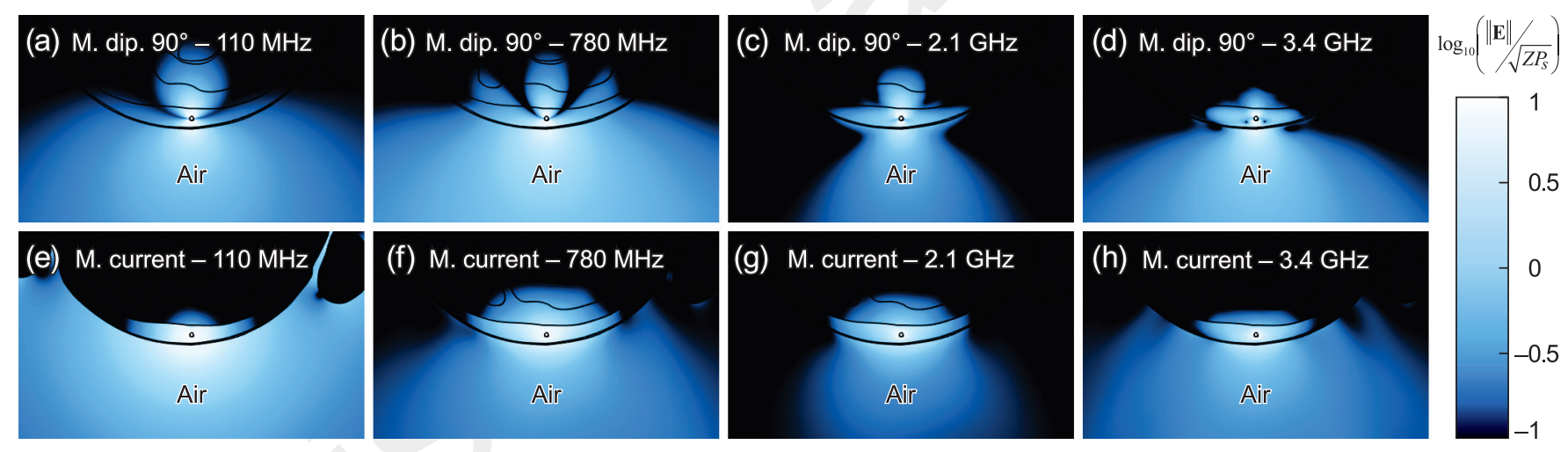

FIG. 11. Normalized E-field distributions of a source at $1 \mathrm{~cm}$ depth (subcutaneous) in anatomical phantom. (a)-(d) The $90^{\circ}$ oriented magnetic dipole moment $\mathbf{m}\left(\mathrm{TM}_{z}\right.$ mode). (e)-(h) The magnetic current source $\left(\mathrm{TE}_{z}\right.$ mode).

rization visibly affects the distribution of $\mathbf{E}$, even though the peak achievable efficiency is within the same range for both sources.

\section{CONCLUSION}

Radiative far- and mid-field EM energy transfer from a body to surrounding free space (where a receiver is located) gives substantially better efficiency than near-field coupling in the $10^{6}-10^{7} \mathrm{~Hz}$ range. For an ideal source, the radiation efficiency increases with frequency until re-
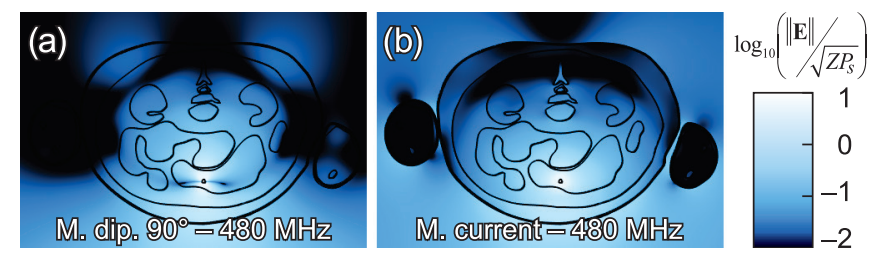

FIG. 12. Normalized E-field distributions of a source at $6 \mathrm{~cm}$ depth (stomach) in anatomical torso phantom at $480 \mathrm{MHz}$. (a) The $90^{\circ}$ oriented magnetic dipole moment $\mathbf{m}\left(\mathrm{TM}_{z}\right.$ mode). (b) The magnetic current source $\left(\mathrm{TE}_{z}\right.$ mode). 
aching its maximum. The frequencies around this maximum are hence optimal; they are inversely proportional to the implantation depth of the source. Maximizing the efficiency for a given depth is negotiating between the attenuation due to the dielectric losses and mismatch losses caused by the high wave-impedance contrast between media. The optimal operating frequency for deep implantation (for instance, ingestible; $d \gtrsim 3 \mathrm{~cm}$ ) is about in the middle of the $10^{8}-10^{9} \mathrm{~Hz}$ range for all four model formulations and can be approximated as $f=2.2 \times 10^{7} / d$. MedRadio $401 \mathrm{MHz}$ and mid-ISM $434 \mathrm{MHz}$ frequency bands belong to this range.

For subcutaneous applications $(d \lesssim 3 \mathrm{~cm})$, the interference effects induced by a standing surface wave are significant. Within the optimal frequency range, the local $\max / \min$ efficiency ratio reaches the value of 6.5 for the realistic case (torso phantom). The exact location of local minima and maxima depends on the implantation depth $d$ as well as skin and subcutaneous fat layers thickness. It is, however, invariant on the source formulation and thus on the polarization. Therefore, for a given subcutaneous device it is important to consider that the optimal frequency depends strongly on the subject and the implantation site. Adaptive frequency hopping within the optimal range may be used to maximize efficiency for subcutaneous applications.

The magnetic dipole (intrinsic directivity $D=1.5$ ) gives about $25 \%$ better efficiency than the isotropic magnetic current source $(D=1)$ for all implantation depths. Lower power dissipation in tissue may be achieved either by increasing the intrinsic antenna directivity to minimize the back lobe radiating inside the body (for fixed position implants) or by beam steering (for implants with undefined positions, e.g. ingestible). Both are applicable only to non-electrically-small antennas.

For the studied depths and frequencies, the waveimpedance contrast between the body and free space accounts for about $80 \%$ to $99 \%$ of radiation efficiency relative loss - in particular, due to total internal reflection. A passive or active on-skin device modulating the surface properties may improve radiation in the far field by mitigating the wave-impedance contrast.

Orienting the source so it primarily radiates using $\mathrm{TE}_{z}$ mode ( $p$-polarization) may improve the transmission efficiency by a few percent, and this effect is inversely proportional to the frequency and depth. However, we observe the opposite for a subcutaneous implantation near curved air-tissue interfaces where fat acts as a matching layer abating reflection losses. Here, $d / \lambda(\omega) \ll 1$, and the problem becomes quasi-static. Radiation efficiency in $f<10^{8} \mathrm{~Hz}$ range rises for the $\mathrm{TM}_{z}$ mode. However, ensuring the minimum realistic bandwidth (for instance, $\mathrm{FBW}_{2}=1 \%$ ) within this range, imposes the limitations on achievable efficiency $\eta_{\mathrm{ub}}$ within the lower part of the optimal frequency range (Figure 10). Yet, this estimate of achievable efficiency $\eta_{\mathrm{ub}}$ for a given bandwidth using (3) gives a conservative value due to the assumptions made (see Section II C). Real-world values would be less restrictive due to re-radiation of energy by currents induced in tissue. These "fringing" currents increase the electrical size of the antenna and therefore raise the physical limitations of achievable (upper bound) radiation efficiency $\eta_{\mathrm{ub}}$ for a given bandwidth (3).

These results establish the optimal frequency range and achievable radiation efficiencies for a far-field energy transfer from magnetic sources inside lossy tissues to surrounding free space. A cautionary remark: even though theoretically possible, a purely magnetic antenna has not been proposed yet. The E-component in the near field of a realistic antenna will unavoidably couple to the tissue leading to increased power dissipation close to the implant. A high-permittivity and low-loss superstrate partially lessens this effect [11].

The findings of this study would not apply if the receiver locates on or near the surface of the body. In that case, the received power can potentially be higher than the radiated power $P_{e}$. The optimal frequency range for a given depth would differ as well for this case [12].

In this study, the established optimal frequency ranges rely on 2D problem formulations. Going beyond this approximation into 3D brings us up against more conditions to consider. Namely: 1) standing surface-wave interference behavior would be more complex affecting the local minima and maxima of the radiation efficiency for shallow sources $(d \lesssim 3 \mathrm{~cm}) ; 2)$ the impure polarizations of radiated EM field; and 3) depending on the application, slightly reduced attenuation losses due to finite $z$-dimensionality. These additional conditions do not influence the main findings of this study in terms of optimal frequency range. Nevertheless, they provide the opening point for further investigations on the maximum achievable radiation efficiency taking into account $3 \mathrm{D}$ surface waves and finite-sized realistic sources.

With proper design of the in-body antennasconsidering the depth of implantation, frequency of operation, radiation modes, polarization, and directivitymore than an order of magnitude improvement of radiation efficiency is feasible compared to existing devices. Such improvement makes it possible to triple the operating range as well as to substantially improve the energy efficiency of miniature wireless body-implants.

\section{ACKNOWLEDGMENTS}

This work was supported by the BodyCap Company (www.bodycap-medical.com), the French Ministry of Foreign Affairs and International Development through the Eiffel Scholarship, the CNRS/DGA through the PEPS program, the Ministry of Education, Youth and Sports of the Czech Republic under the RICE - New Technologies and Concepts for Smart Industrial Systems, project No. LO1607. D. N. thanks F. Foglia Manzillo (CEA Leti) and I. Iliopoulos (IETR) for discussions. 


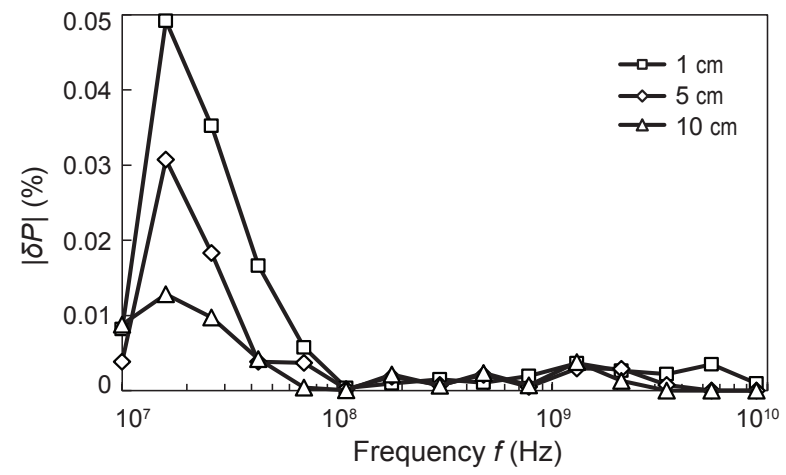

FIG. 13. Absolute value of the relative residual power error for infinite planar-bounded half-space homogeneous medium.

\section{Appendix A: Numerical Model}

The formulated problem requires a numerical solution of the second-order linear partial differential equation (4). We use the $h p$-FEM method implemented into the inhouse code Agros2D [30] as it gives more accurate representation of curved interfaces for the same mesh density compared, for instance, to FDTD. The results obtained for each geometry are validated using the FEM solver of COMSOL Multiphysics ${ }^{\circledR} 5.2$ [31]. For the planar homogeneous half-space medium, we additionally verify the results using the FDTD method implemented in Meep [32]. The results agree within the $\delta \eta_{\text {idl }}<1 \%$ tolerance for each studied frequency and depth.
The frequency range spans from $10^{7} \mathrm{~Hz}$ to $10^{10} \mathrm{~Hz}$ and is discretized into $16 \log _{10}$-spaced intervals. For the planar homogeneous and layered heterogeneous models, a circular perfectly matched layer (PML) truncates the study domain at the distance $2 \lambda\left(\varepsilon_{0}\right)$ from the source to ensure that negligibly small power reaches the tissuePML boundary. For the circular and realistic abdominal models, the distance to the PML is $\lambda$ from the models. The exiting power $P_{e}(7)$ is evaluated on the inner boundary of the PML. The Dirichlet boundary condition $\eta \times \mathbf{E}=0$ (perfect electric conductor) is imposed the outside of the PML. The initial mesh contains first order elements with the maximum length $\lambda(\varepsilon) / 4$ for each analyzed frequency. The automatic $h p$-adaptivity algorithm selectively refines specific elements and operates up to ninth polynomial order. The accuracy is set to maintain the total energy relative error below $0.5 \%$.

\section{Appendix B: Conservation of Energy}

Conservation of energy (power balance) is one of the necessary conditions of the model validity. To verify this condition, we calculated the relative residual power error as $\delta P=\left(P_{s}-P_{e}-P_{d}\right) / P_{s}$ for every solution given here. According to the numerical model formulation, we consider the model valid when $\delta P<0.5 \% \forall \eta_{\text {idl }}(f, d)$. The planar model formulations give the highest error due to the PML-truncation of the half-space representing tissue. Figure 13 shows the relative residual power errors $\delta P$ for the planar homogeneous medium. The maximum error $\max |\delta P| \ll 0.5 \%$ at $d=1 \mathrm{~cm}, f=1.6 \times 10^{7} \mathrm{~Hz}$.
[1] A. Kiourti and K. S. Nikita, "A review of in-body biotelemetry devices: implantables, ingestibles, and injectables," IEEE Trans. Biomed. Eng. 64, 1422-1430 (2017).

[2] John S. Ho, Yuji Tanabe, Shrivats Mohan Iyer, Amelia J. Christensen, Logan Grosenick, Karl Deisseroth, Scott L. Delp, and Ada S.Y. Poon, "Self-tracking energy transfer for neural stimulation in untethered mice," Phys. Rev. Applied 4, 024001 (2015).

[3] Taeil Kim et al., "Injectable, cellular-scale optoelectronics with applications for wireless optogenetics," Science 340, 211-216 (2013).

[4] Sanghoek Kim, John S. Ho, and Ada S. Y. Poon, "Midfield wireless powering of subwavelength autonomous devices," Phys. Rev. Lett. 110, 203905 (2013).

[5] Sanghoek Kim, John S. Ho, Lisa Y. Chen, and Ada S. Y. Poon, "Wireless power transfer to a cardiac implant," Appl. Phys. Lett. 101, 073701 (2012).

[6] Devansh R. Agrawal, Yuji Tanabe, Desen Weng, Andrew Ma, Stephanie Hsu, Song-Yan Liao, Zhe Zhen, Zi-Yi Zhu, Chuanbowen Sun, Zhenya Dong, Fengyuan Yang, Hung Fat Tse, Ada S. Y. Poon, and John S. Ho, "Conformal phased surfaces for wireless powering of bioelectronic microdevices," Nat. Biomed. Eng. 1, 0043 (2017).
[7] John S. Ho, Alexander J. Yeh, Evgenios Neofytou, Sanghoek Kim, Yuji Tanabe, Bhagat Patlolla, Ramin E. Beygui, and Ada S. Y. Poon, "Wireless power transfer to deep-tissue microimplants," PNAS 111, 7974-7979 (2014).

[8] Camelia Gabriel, S. Gabriel, and E. Corthout, "The dielectric properties of biological tissues: I. Literature survey," Phys. Med. Biol. 41, 2231-2249 (1996), 11.

[9] S. Gabriel, R. W. Lau, and Camelia Gabriel, "The dielectric properties of biological tissues: II. Measurements in the frequency range $10 \mathrm{~Hz}$ to $20 \mathrm{GHz}$," Phys. Med. Biol. 41, 2251-2269 (1996).

[10] S. Gabriel, R. W. Lau, and Camelia Gabriel, "The dielectric properties of biological tissues: III. Parametric models for the dielectric spectrum of tissues," Phys. Med. Biol. 41, 2271-2293 (1996).

[11] Denys Nikolayev, Maxim Zhadobov, Laurent Le Coq, Pavel Karban, and Ronan Sauleau, "Robust ultraminiature capsule antenna for ingestible and implantable applications," 65, 6107-6119 (2017), IEEE Trans. Antennas Propag.

[12] Ada S. Y. Poon, Stephen O'Driscoll, and Teresa H. Meng, "Optimal frequency for wireless power transmission into dispersive tissue," IEEE Trans. Antennas Pro- 
pag. 58, 1739-1750 (2010)

[13] Denys Nikolayev, Maxim Zhadobov, Ronan Sauleau, and Pavel Karban, "Antennas for ingestible capsule telemetry," in Advances in body-centric wireless communication: Applications and state-of-the-Art (IET, London, UK, 2016) pp. 143-186.

[14] M. Hendriks M. J. Peters, G. Stinstra, "Estimation of the electrical conductivity of human tissue," Electromagnetics 21, 545-557 (2001).

[15] Camelia Gabriel, "Dielectric properties of biological materials," in Handbook of biological effects of electromagnetic fields. Bioengineering and biophysical aspects of electromagnetic fields, edited by Frank S. Barnes and Ben Greenebaum (CRC Press, Boca Raton, FL, 2007) 3rd ed., pp. 51-100.

[16] Alexander Puzenko, Paul Ben Ishai, and Yuri Feldman, "Cole-Cole broadening in dielectric relaxation and strange kinetics," Phys. Rev. Lett. 105, 037601 (2010).

[17] Akimasa Hirata, Sachiko Kodera, Jianqing Wang, and Osamu Fujiwara, "Dominant factors influencing wholebody average SAR due to far-field exposure in wholebody resonance frequency and $\mathrm{GHz}$ regions," Bioelectromagnetics 28, 484-487 (2007).

[18] Francesco Merli, B. Fuchs, J. R. Mosig, and A. K. Skrivervik, "The effect of insulating layers on the performance of implanted antennas," IEEE Trans. Antennas Propag. 59, 21-31 (2011).

[19] D. P. Chrissoulidis and J. M. Laheurte, "Radiation from an encapsulated hertz dipole implanted in a human torso model," IEEE Trans. Antennas Propag. 64, 4984-4992 (2016).

[20] C. A. Balanis, Advanced engineering electromagnetics, 2nd ed. (John Wiley \& Sons, Hoboken, NJ, 2012).
[21] Frank S. Barnes and Ben Greenebaum, Handbook of biological effects of electromagnetic fields. Bioengineering and biophysical aspects of electromagnetic fields, 3rd ed. (CRC/Taylor \& Francis, Boca Raton, 2007).

[22] H.A. Wheeler, "Fundamental limitations of small antennas," Proc. IRE 35, 1479-1484 (1947).

[23] L. J. Chu, "Physical limitations of omni-directional antennas," J. Appl. Phys. 19, 1163-1175 (1948).

[24] J. S. McLean, "A re-examination of the fundamental limits on the radiation Q of electrically small antennas," IEEE Trans. Antennas Propag. 44, 672-676 (1996).

[25] Constantine A. Balanis, Antenna theory: analysis and design, 4th ed. (John Wiley \& Sons, Hoboken, NJ, 2016).

[26] S. R. Best and A. D. Yaghjian, "The lower bounds on Q for lossy electric and magnetic dipole antennas," IEEE Antenn. Wireless Propag. Lett. 3, 314-316 (2004).

[27] A. D. Yaghjian and S. R. Best, "Impedance, bandwidth, and Q of antennas," IEEE Trans. Antennas Propag. 53, 1298-1324 (2005).

[28] Dhiraj Sinha and Gehan A.J. Amaratunga, "Electromagnetic radiation under explicit symmetry breaking," Phys. Rev. Lett. 114, 147701 (2015).

[29] A. Christ, T. Samaras, A. Klingenbock, and N. Kuster, "Characterization of the electromagnetic near-field absorption in layered biological tissue in the frequency range from $30 \mathrm{MHz}$ to 6,000 MHz," Phys. Med. Biol. 51, 49514965 (2006).

[30] Pavel Karban, Frantisek Mach, Pavel Kus, David Panek, and Ivo Dolezel, "Numerical solution of coupled problems using code Agros2d," Computing 95, 381-408 (2013).

[31] COMSOL Inc., "COMSOL Multiphysics," (2017).

[32] Ardavan F. Oskooi, David Roundy, Mihai Ibanescu, Peter Bermel, J. D. Joannopoulos, and Steven G. Johnson, "Meep: A flexible free-software package for electromagnetic simulations by the FDTD method," Comput. Phys. Commun. 181, 687-702 (2010). 